\title{
The Nutritional Status of Cook Islanders
}

\author{
By S. FAINE AND C. E. HERCUS \\ Departments of Bacteriology and Preventive Medicine, University of \\ Otago Medical School, Nero Zealand
}

(Received 2 fanuary 1951)

Several observers have noted profound changes in the diet of the Rarotongan since the advent of the European about roo years ago (Davis, 1947; Abraham, 1947; Peren, 1947; Wingfield, 1949).

Although native local foods still remain the staple diet, increasing reliance is being placed on imported European-type food, chiefly meat, flour, sugar and biscuits. With a view towards assessing the effects of these changes and the adequacy of the diet as a whole, nutritional status was investigated in the course of a survey of the health of Cook Islanders carried out in January and February $195^{\circ}$.

\section{METHODS}

The subjects of this survey were 365 people of all ages, chosen at random in sixty-six family groups from the village of Arorangi, on the island of Rarotonga, in the Cook Islands. As seen from Table I, they comprised a representative sample (about onethird) of the population of the village of Arorangi, and in turn a representative sample of the whole island's population of approximately 6000 .

Table I. Age and sex distribution of subjects of survey and of population of Arorangi village and of Island of Rarotonga

\begin{tabular}{|c|c|c|c|c|c|c|c|c|c|c|c|c|c|}
\hline \multirow{3}{*}{$\begin{array}{c}\text { Age } \\
\text { (years) }\end{array}$} & \multicolumn{6}{|c|}{ Subjects of survey $195^{\circ}$} & \multicolumn{6}{|c|}{ Arorangi I 945 census } & \multirow{3}{*}{$\begin{array}{c}\text { Rarotonga } \\
\text { r945 } \\
\text { census } \\
(\%)\end{array}$} \\
\hline & \multicolumn{2}{|c|}{ Male } & \multicolumn{2}{|c|}{ Female } & \multicolumn{2}{|c|}{ Total } & \multicolumn{2}{|c|}{ Male } & \multicolumn{2}{|c|}{ Female } & \multicolumn{2}{|c|}{ Total } & \\
\hline & No. & $\%$ & No. & $\%$ & No. & $\%$ & No. & $\%$ & No. & $\%$ & No. & $\%$ & \\
\hline Under 5 & 26 & $14 \cdot 2$ & 29 & I $5 \cdot 9$ & 5.5 & $15 \cdot I$ & 88 & $15 \cdot 1$ & 96 & $17 \cdot 9$ & 184 & $16 \cdot 5$ & $15 \cdot 9$ \\
\hline $5-9$ & 22 & $12 \cdot 0$ & 34 & 18.7 & 56 & $15 \%$ & 118 & $20 \cdot 3$ & 106 & $19 \cdot 6$ & 224 & 19.9 & $15 \cdot 7$ \\
\hline $10-14$ & 4 I & $22 \cdot 4$ & 34 & $18 \cdot 7$ & 75 & $20 \cdot 6$ & 109 & 18.7 & 84 & $15 \cdot 7$ & 193 & $17 \cdot 2$ & 13.6 \\
\hline $15-19$ & 24 & $13 \cdot 1$ & 12 & $6 \cdot 6$ & 36 & $9 \cdot 8$ & 58 & $10 \cdot 1$ & 42 & & 100 & 8.9 & $9 \cdot 8$ \\
\hline $20-29$ & rs & $8 \cdot 2$ & 20 & II $\cdot 0$ & 35 & $9 \cdot 6$ & 69 & $11 \cdot 9$ & 73 & $13 \cdot 6$ & 142 & $12 \cdot 3$ & 15.0 \\
\hline $30-39$ & 27 & 14.8 & $2 I$ & $11 \cdot 6$ & $4^{8}$ & $13 \cdot 2$ & 55 & 9.5 & 55 & 10.4 & IIo & 10.0 & 10.9 \\
\hline $40-49$ & 12 & $6 \cdot 5$ & I 6 & 8.8 & 28 & $7 \cdot 6$ & 32 & $5 \cdot 5$ & 27 & $5^{\cdot 1}$ & 59 & $5 \cdot 3$ & $8 \cdot 3$ \\
\hline $50-59$ & Io & $5 \cdot 5$ & I I & 6.0 & 21 & 5.7 & 28 & $4 \cdot 8$ & 24 & $4 \cdot 5$ & 52 & $4 \cdot 6$ & 6.0 \\
\hline $\begin{array}{l}60 \text { and } \\
\text { over }\end{array}$ & 6 & $3 \cdot 3$ & 5 & $2 \cdot 7$ & I I & $3 \cdot 0$ & 24 & $4^{\prime} 1$ & 29 & $5 \cdot 4$ & 51 & $4 \cdot 7$ & $4 \cdot 8$ \\
\hline Total & 183 & $100 \cdot 0$ & 182 & 100.0 & $36_{5}$ & 100.0 & 581 & 100.0 & 536 & $100 \cdot 0$ & I I I 7 & 100.0 & 一 \\
\hline
\end{tabular}

By occupation, fifty of the adults were planters entirely dependent upon the products of their plantation, thirty-one worked regularly for wages in addition to planting, and twenty-one were entirely dependent upon wages which averaged about 6 s. a day. Of 
the group, $67 \%$ were full Polynesian, $\times 5 \%$ from a quarter to half European, and Ir. $5 \%$ half or more European.

There is no satisfactory single index of nutritional status. Methods that measure 'nutritional status', or any or some of its various components, may be either subjective or objective or both.

\section{Clinical methods}

The methods used included a general clinical examination and an assessment ('subjective nutritional assessment') arrived at by roughly averaging marks (ascending from I to 5 with improving condition) for each of skeletal development, muscular development and tone, quality and quantity of subcutaneous tissue, skin condition and general appearance. Marks were deducted for each defect from a possible 5 in each category, according to a definite scale.

\section{Dental state}

A full dental examination was made and the condition of the teeth recorded according to a modification of the Medical Research Council system (Mellanby, 1934).

\section{Anthropometric measurements}

Objective measurements of various components of nutritional status were made as follows:

Height was measured without shoes to the nearest 1 in.

Weight was taken on a beam balance without shoes but with clothes (always very light), in $\mathrm{lb}$.

The weight: height ratio $(W: H)$ was calculated as weight in $\mathrm{lb}$. divided by the height in inches.

The Tuxford index was calculated after Tuxford (1942) from $\frac{W}{H} \times \frac{336-m}{270}$ for boys, and $\frac{W}{H} \times \frac{308-m}{235}$ for girls, where $m$ is the age in months.

\section{Laboratory tests}

Haemoglobin was measured both colorimetrically by the Hellige apparatus modification of Sahli's method, and by calculation from blood specific gravity (Phillips, Van Slyke, Dole, Emerson, Hamilton \& Archibald, 1945; Van Slyke, Phillips, Dole, Hamilton, Archibald \& Plazin, I950).

Erythrocytes were counted by standard methods.

Plasma protein was measured in oxalated plasma by the copper-sulphate specific gravity method of Phillips et al. (1945) and of Van Slyke, Hiller, Phillips, Hamilton, Dole, Archibald \& Eder (1950). The formula,

$$
\text { protein }(\mathrm{g} . / 100 \mathrm{ml} .)=364\left(G_{p}-\mathrm{r} \cdot 006\right) \text {, }
$$

where $G_{p}$ is the observed specific gravity, was used, following Hoch \& Marrack (1945). 
This gives values for protein approximately $0.15 \mathrm{~g} . / 100 \mathrm{ml}$. higher over the range, than the formula, $373\left(G_{p}-\mathrm{I} \cdot 007\right)$, recommended by Van Slyke, Phillips et al. (1950).

Sedimentation rate was measured by Wintrobe's method (Wintrobe, 1935).

\section{Dietary assessment}

A qualitative assessment of the diet of each family was made by interrogation and observation.

\section{Vital statistics}

The vital statistics for the Cook Islands as a whole and for Rarotonga were considered.

\section{Clinical methods}

\section{RESULTS}

General physical examination. On the whole the people appeared reasonably clean, cheerful and well nourished. Almost without exception their co-operation was good. There was one case of malnutrition, in an infant of 8 months, following a bout of unspecified diarrhoea several weeks previously. The Mantoux test was negative, but the home conditions and parental education were among the poorest seen. The only deformities seen were due to trauma, or had some explanation other than malnutrition. No clinical signs of malnutrition were noted. Only one case of thyroid enlargement of a minor nature was seen.

Skin conditions. Scars, of both infective and traumatic origin, were encountered almost universally; scabies, head-lice and ringworm were also common. No skins were seen that reflected nutritional deficiency.

Table 2. Subjective nutritional assessment of subjects of survey based on assignment of marks from I for poor to 5 for excellent for each of skeletal development, muscular development and bone, quality and quantity of subcutaneous tissue, skin condition and general appearance

\begin{tabular}{|c|c|c|c|c|c|c|c|c|c|c|c|}
\hline \multirow{3}{*}{$\begin{array}{c}\text { Age } \\
\text { (years) }\end{array}$} & \multirow{3}{*}{$\begin{array}{c}\text { No. } \\
\text { examined }\end{array}$} & \multicolumn{10}{|c|}{ Marks } \\
\hline & & \multicolumn{2}{|c|}{ I } & \multicolumn{2}{|c|}{2} & \multicolumn{2}{|c|}{3} & \multicolumn{2}{|c|}{4} & \multicolumn{2}{|c|}{5} \\
\hline & & No. & $\%$ & No. & $\%$ & No. & $\%$ & No. & $\%$ & No. & $\%$ \\
\hline $0-2$ & 34 & $\mathbf{I}$ & $3 \cdot 2$ & 8 & 23.5 & 14 & $4 I \cdot I$ & II & $32 \cdot 2$ & 一 & - \\
\hline $3-5$ & 32 & - & - & 3 & 9.4 & 13 & 40.6 & 16 & $50 \cdot 0$ & - & - \\
\hline $6-12$ & 90 & - & - & 6 & $6 \cdot 6$ & 49 & $54: 4$ & 35 & $38 \cdot 8$ & - & - \\
\hline $13-20$ & 68 & - & - & 4 & $6 \cdot 5$ & 19 & $27 \cdot 9$ & 44 & 64.7 & I & $I \cdot 4$ \\
\hline $21-45$ & 98 & I & $1 \cdot 0$ & 4 & $4^{\circ} 0$ & 43 & $43 \cdot 8$ & 49 & $50 \cdot 0$ & 一 & - \\
\hline Over 45 & 43 & I & $2 \cdot 3$ & 6 & 13.9 & 23 & $53 \cdot 5$ & 13 & $30 \cdot 2$ & - & - \\
\hline Total & $3^{6} 5$ & 3 & 0.8 & $3 x$ & $8 \cdot 2$ & 161 & $44 \cdot I$ & 168 & $46 \cdot 0$ & I & 0.3 \\
\hline
\end{tabular}

Subjective nutritional assessment. The results are shown in Table 2. It will be seen that most people are graded 3-4, that is, average to good. Only one person was so outstanding as to deserve 5, and only three so poor as to deserve I; two of these suffered disability following accidents. Posture was remarkably good, as was muscular 
development. Obesity was the rule, especially in women, as seen from the heightweight graphs (see below).

\section{Dental and oral examination}

The results of the dental examination are shown in Tables 3 and 4 . It will be seen that of the $36 \mathrm{I}$ mouths examined, twenty-four were edentulous, eight on account of infancy, sixteen had had all their teeth extracted and of these nine had no dentures. Of the remaining 337 , twelve $(4 \%)$ were free from dental caries (ten of these were I year old or less, one was aged 3 , and one 15 ). Of the 325 with dental caries, 258 $(80 \%)$ were over 6 years of age. The table shows that there was no important difference between the incidence in the various age groups.

The degree of caries and the number of fillings are shown in Tables 3 and 4 by age groups. It is evident that the severity of the caries increases as age advances. It is noteworthy that only one individual over 40 had any fillings; this man had been overseas with the Cook Island contingent to the first World War and the copperamalgam fillings were the work of the dental unit at Moascar Camp. Most of the dental work had been carried out by the School Dental Service but in few mouths had the necessary dental work been completed. Out of sixteen totally edentulous people seven had full dentures, and two out of nine without upper teeth had full upper plates and six others had partial upper plates. The same type of anomalies was found as was mentioned by Wingfield (1949). This hypoplasia of enamel was found in a number of cases. In one family all members were affected. A number of cases of cracked enamel on the palatal surfaces of the upper incisors was noted, and marked attrition on the occlusal surfaces of the molars with exposure of dentine-such teeth were free of caries. The use of a toothbrush is being introduced among schoolchildren.

Health of gums. The incidence of gingival disease by age groups in 343 individuals in this study contrasted with the number of teeth present in the mouth is shown in Table 5. The steadily increasing amount and degree of gingival disease with advancing age is evident.

Health of pharynx. Table 6 shows that the state of health of the throat and tonsils was excellent.

\section{Anthropometric measurements}

Height and weight. The average height and weight for each age group are shown in Tables 7 and 8 ; in addition, the weights in relation to height, for males and females irrespective of age, are shown in Fig. I. The heights and weights of Rarotongan children studied in this group are compared with similar figures for children of comparable age from other countries in Tables 9 and 10.

In an attempt to standardize figures to a comparable base, weight: height $(W: H)$ ratios and the Tuxford index (Tuxford, 1942) for the groups shown in Tables 9 and ro have been calculated and are presented in 'Table i I and Fig. 2. It will be seen from all these figures that, although the samples are small, Rarotongan children are of approximately the same build, height and weight as New Zealand European children, smaller than New Zealand Maoris, but bigger than the other groups in most respects. 


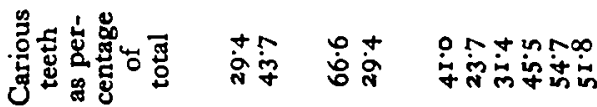

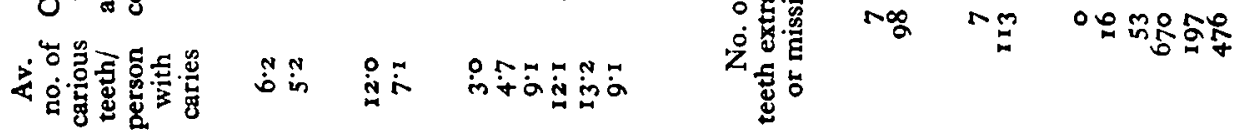

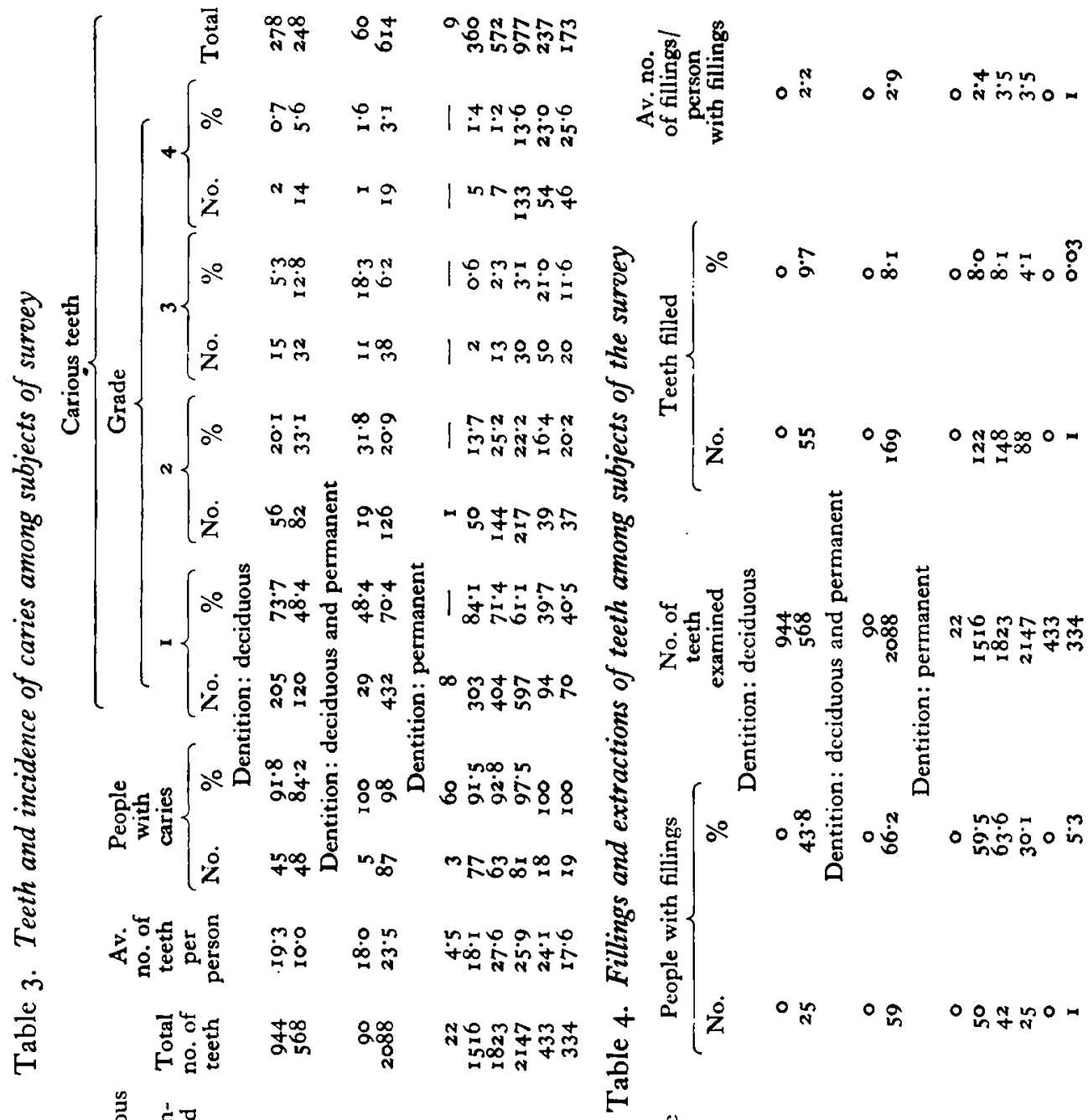

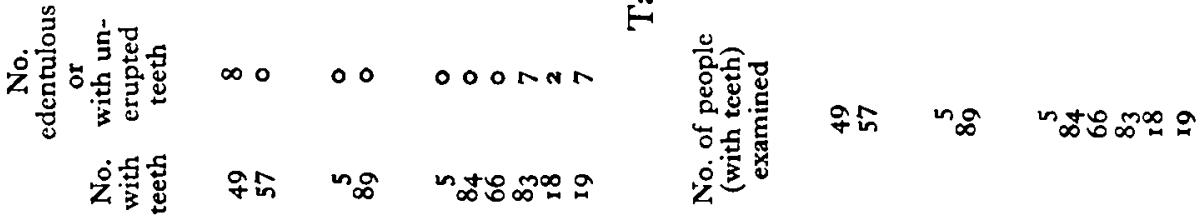

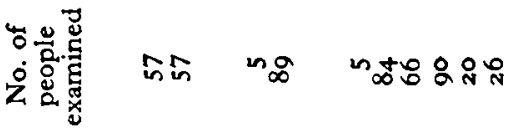
镡

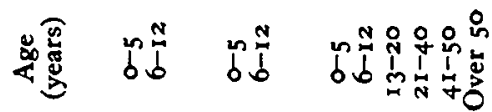




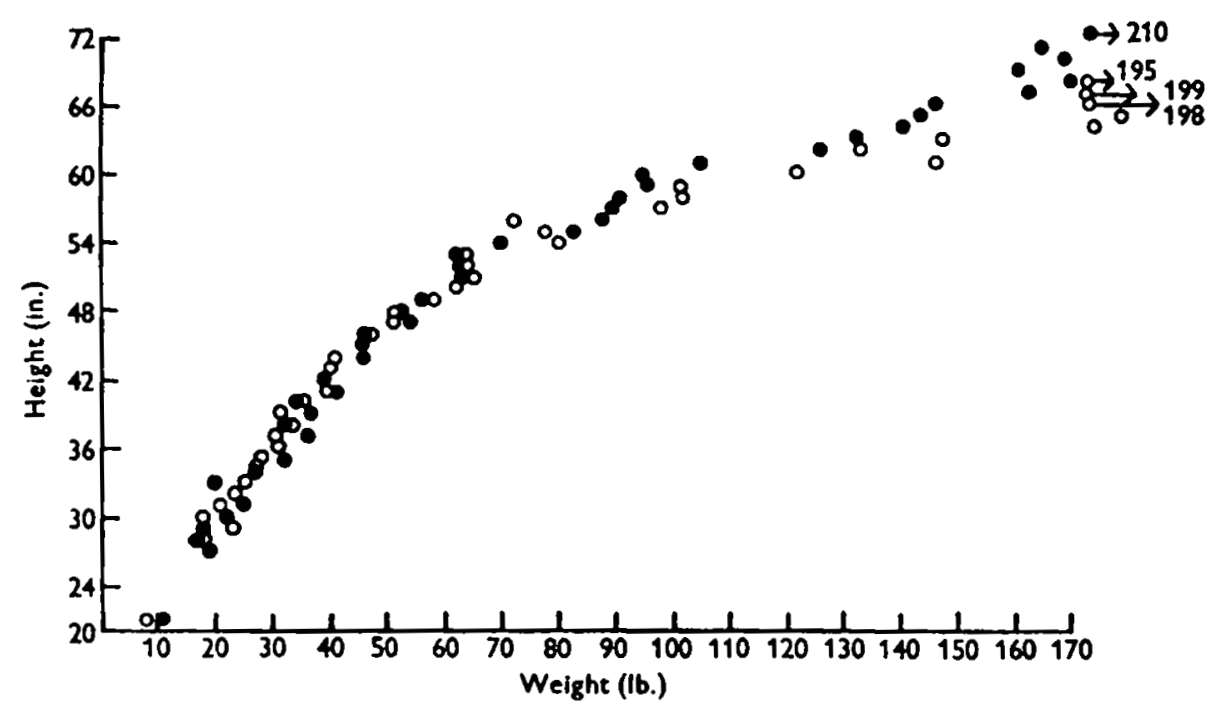

Fig. 1. Average weight in lb. of sample of population of Arorangi, classified according to height in inches. males; $O$, females.

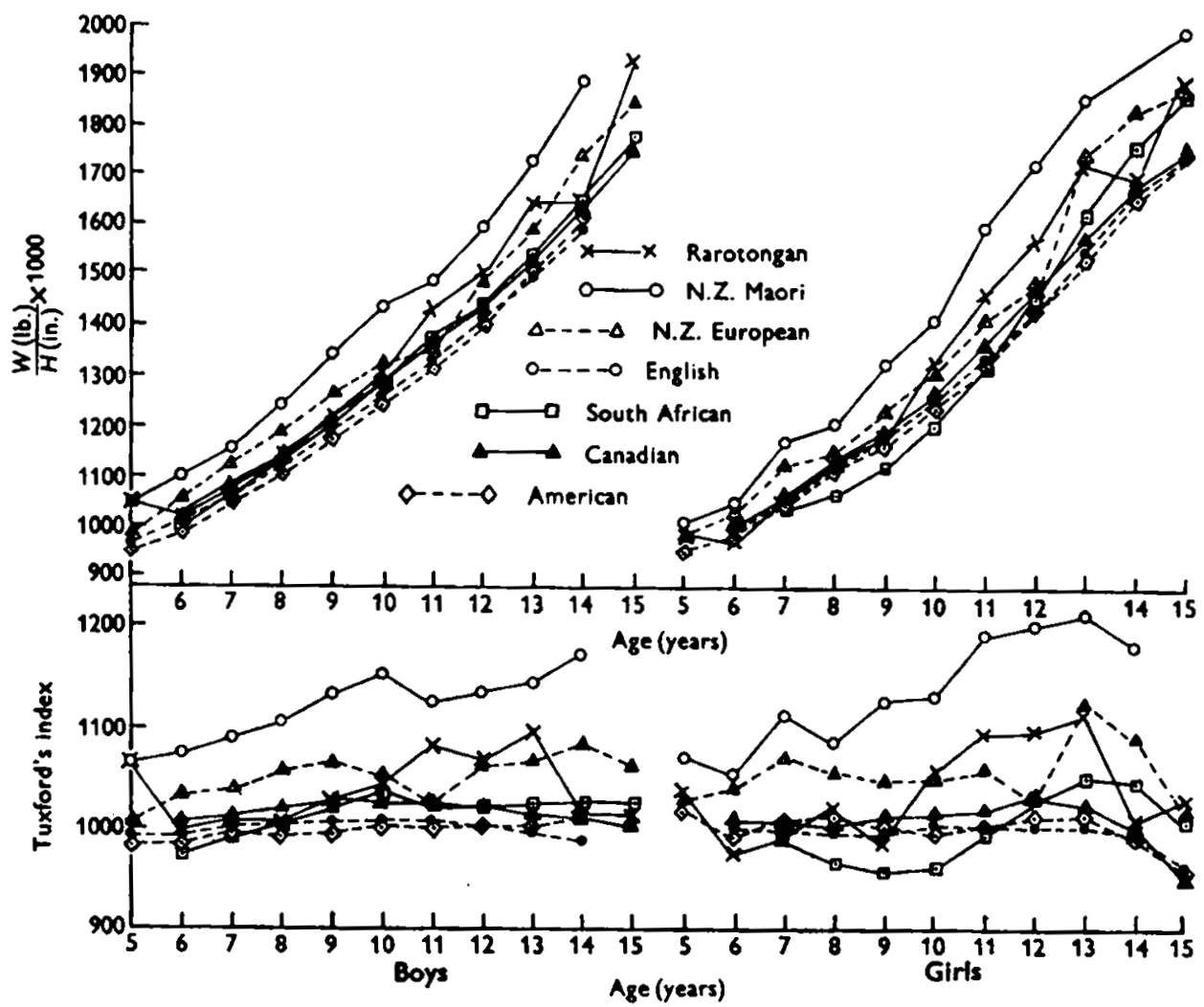

Fig. 2. Weight: height ratio and Tuxfond's index for children measured on Rarotong*, classified according to age and compared with published data for children of other races. 
Table 5. Condition of gums of subjects of survey

\begin{tabular}{|c|c|c|c|c|c|c|c|c|c|c|c|c|c|}
\hline \multirow{3}{*}{$\begin{array}{c}\text { Age } \\
\text { (years) }\end{array}$} & \multirow{3}{*}{$\begin{array}{c}\text { No. of } \\
\text { people } \\
\text { (with teeth) } \\
\text { examined }\end{array}$} & \multicolumn{12}{|c|}{ People with gingivitis in grade* } \\
\hline & & \multicolumn{2}{|c|}{ o } & \multicolumn{2}{|c|}{ I } & \multicolumn{2}{|c|}{2} & \multicolumn{2}{|c|}{3} & \multicolumn{2}{|c|}{4} & \multicolumn{2}{|c|}{$s$} \\
\hline & & No. & $\%$ & No. & $\%$ & No. & $\%$ & No. & $\%$ & No. & $\%$ & No. & $\%$ \\
\hline $\begin{array}{l}0-5 \\
6-12\end{array}$ & 66 & $\begin{array}{l}62 \\
80\end{array}$ & $\begin{array}{l}93.9 \\
88.0\end{array}$ & 3 & 45 & $x$ & 1.5 & - & 一 & - & 一 & - & - \\
\hline $\begin{array}{r}12-20 \\
\end{array}$ & 66 & 47 & $\begin{array}{l}00 \cdot 9 \\
7.2\end{array}$ & 9 & 1000 & I & $\begin{array}{lll}1.1 \\
0.7\end{array}$ & $\overline{2}$ & $\overline{1.5}$ & - & - & - & 一 \\
\hline $21-40$ & $8_{3}$ & 12 & 14.5 & 23 & 27.8 & 22 & $26 \cdot 4$ & 21 & $\begin{array}{r}4.3 \\
25.3\end{array}$ & 6 & $7 \cdot 3$ & - & 一 \\
\hline $41-50$ & 18 & ○ & 0 & 4 & $22 \cdot 2$ & 6 & $33 \cdot 3$ & 5 & 27.8 & 2 & Ir. I & $\mathrm{I}$ & 5.5 \\
\hline Over $5^{\circ}$ & 20 & I & $5 \cdot 0$ & o & - & 4 & $20 \cdot 0$ & 7 & $35^{\circ} 0$ & 5 & $25^{\circ} 0$ & 3 & 15.0 \\
\hline Total & 343 & - & 一 & - & - & - & - & 一 & - & - & - & - & - \\
\hline
\end{tabular}

- 0 : gums healthy; 1 : commencing gingivitis; 2 and 3 : progressively increasing gingivitis and early pyorrhoea; 4 and $5:$ severe and gross pyorrhoea.

Table 6. Condition of throat and tonsils of subjects of survey graded in age groups

\begin{tabular}{|c|c|c|c|c|c|c|c|}
\hline \multirow{3}{*}{$\begin{array}{c}\text { Age } \\
\text { (years) }\end{array}$} & \multirow{3}{*}{$\begin{array}{l}\text { Total no. } \\
\text { examined }\end{array}$} & \multicolumn{6}{|c|}{ Grading* } \\
\hline & & \multicolumn{2}{|c|}{0} & \multicolumn{2}{|c|}{ I } & \multicolumn{2}{|c|}{2} \\
\hline & & No. & $\%$ & No. & $\%$ & No. & $\%$ \\
\hline $0-5$ & 66 & 58 & 88.0 & 6 & $9 \cdot 1$ & 2 & 3.0 \\
\hline $6-12$ & 90 & 74 & $82 \cdot 3$ & 12 & $13 \cdot 3$ & 4 & $4 \cdot 4$ \\
\hline $13-20$ & 66 & 54 & $81 \cdot 7$ & 10 & 15.3 & 2 & 3.0 \\
\hline $21-40$ & 90 & 79 & $87 \cdot 8$ & 11 & 12.2 & - & - \\
\hline $41-50$ & 20 & 18 & $90 \cdot 0$ & 2 & 10.0 & - & - \\
\hline Over 50 & 29 & 25 & $86 \cdot 2$ & 4 & 13.8 & - & - \\
\hline
\end{tabular}

*: healthy throat and tonsils; $\mathrm{I}$ : enlargement of tonsils; 2 : enlarged tonsils and infected throat. No cases of tonsillar enlargement or unhealthy throats beyond this degree were recorded.

Table 7. Ages and heights of subjects of the survey

\begin{tabular}{|c|c|c|c|c|c|c|c|c|c|c|c|c|c|c|c|}
\hline \multirow{4}{*}{$\begin{array}{c}\text { Age } \\
\text { (years) }\end{array}$} & \multicolumn{8}{|c|}{ Males } & \multicolumn{7}{|c|}{ Females } \\
\hline & \multirow{3}{*}{$\begin{array}{c}\text { No. } \\
\text { examined }\end{array}$} & \multicolumn{7}{|c|}{ Height } & \multirow{3}{*}{$\begin{array}{c}\text { No. } \\
\text { examined }\end{array}$} & \multicolumn{6}{|c|}{ Height } \\
\hline & & \multirow{2}{*}{\multicolumn{2}{|c|}{$\begin{array}{c}\text { Average } \\
\text { ft. in. }\end{array}$}} & \multicolumn{5}{|c|}{ Range } & & Average & \multicolumn{5}{|c|}{ Range } \\
\hline & & & & ft. & in. & & ft. & in. & & ft. in. & ft. & in. & & ft. & in. \\
\hline 2 & 5 & 3 & 0 & 2 & 7 & to & 3 & $I$ & 5 & $210\}$ & 2 & 8 & to & 3 & 3 \\
\hline 3 & 4 & 3 & 0 & 2 & 9 & to & 3 & 3 & 7 & 3 I & 2 & 9 & to & 3 & 5 \\
\hline 4 & 5 & 3 & 3 & 3 & $\mathbf{I}$ & to & 3 & 4 & 4 & 34 & 3 & 4 & to & 3 & 5 \\
\hline 5 & 6 & 3 & 6 & 3 & $\mathbf{I}$ & to & 3 & 9 & 5 & $34 \frac{1}{2}$ & 3 & 4 & to & 3 & 5 \\
\hline 6 & 4 & 3 & $7 \frac{1}{2}$ & 3 & 5 & to & 3 & II & 13 & 39 & 3 & 7 & to & 4 & I \\
\hline 7 & 3 & 4 & 0 & 3 & 10 & to & 4 & I & 6 & $3 \mathrm{rot}$ & 3 & 9 & to & 4 & $\mathbf{I}$ \\
\hline 8 & 6 & 4 & of & 3 & 10 & to & 4 & 4 & 3 & 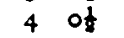 & 4 & 0 & to & 4 & I \\
\hline 9 & 3 & 4 & $4 \frac{1}{2}$ & 4 & 3 & to & 4 & 4 & 6 & 42 & 4 & 0 & to & 4 & 3 \\
\hline ro & 8 & 4 & 4 & 4 & $\mathbf{I}$ & to & 4 & 7 & 7 & 45 & 4 & 3 & to & 4 & 10 \\
\hline I I & 6 & 4 & $7 t$ & 4 & 4 & to & 4 & ro & 10 & 471 & 4 & I & to & 5 & 0 \\
\hline 12 & ro & 4 & $8 t$ & 4 & 4 & to & 5 & 2 & 4 & $410 \frac{z}{7}$ & 4 & 5 & to & 5 & $\mathbf{I}$ \\
\hline 13 & 6 & 5 & 0 & 5 & I & to & 5 & 3 & 8 & $4 \mathrm{II}$ & 4 & 4 & to & 5 & 3 \\
\hline 14 & IO & 5 & 4 & 4 & 10 & to & 5 & 4 & 5 & 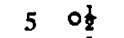 & 4 & 10 & to & 5 & 4 \\
\hline I 5 & 9 & 5 & 4 & 5 & 0 & to & 5 & 6 & 2 & $5 \quad 1 \frac{1}{2}$ & 4 & I I & to & 5 & 4 \\
\hline 16 & 4 & 5 & 7 & 5 & 5 & to & 5 & 9 & 2 & $5 \quad 3 \frac{1}{2}$ & 5 & 3 & to & 5 & 4 \\
\hline Over 16 & 82 & 5 & $6 \frac{1}{8}$ & 5 & I & to & 6 & 0 & 81 & 53 & 4 & 9 & to & 5 & 8 \\
\hline Total & I7I & - & & & & 一 & & & 168 & - & & & - & & \\
\hline
\end{tabular}


Table 8. Ages and weights of subjects of the survey

\begin{tabular}{|c|c|c|c|c|c|c|}
\hline \multirow[b]{3}{*}{$\begin{array}{l}\text { Age } \\
\text { (years) }\end{array}$} & \multicolumn{3}{|c|}{ Males } & \multicolumn{3}{|c|}{ Females * } \\
\hline & \multirow[b]{2}{*}{$\begin{array}{l}\text { No. } \\
\text { examined }\end{array}$} & \multicolumn{2}{|c|}{ Weight } & \multirow[b]{2}{*}{$\begin{array}{l}\text { No. } \\
\text { examined }\end{array}$} & \multicolumn{2}{|c|}{ Weight } \\
\hline & & $\begin{array}{c}\text { Average } \\
\text { (lb.) }\end{array}$ & $\begin{array}{c}\text { Range } \\
\text { (lb.) }\end{array}$ & & $\begin{array}{c}\text { Average } \\
\text { (lb.) }\end{array}$ & $\begin{array}{c}\text { Range } \\
\text { (lb.) }\end{array}$ \\
\hline Under $\frac{1}{2}$ & 4 & $15 \cdot 3$ & $11 \cdot 2-18 \cdot 2$ & 4 & 130 & $8 \cdot 4-18 \cdot 2$ \\
\hline$\frac{1}{2}-1$ & 0 & - & & 3 & $18 \cdot 2$ & $16.8-19.6$ \\
\hline 1 & 5 & $20 \cdot 2$ & $16 \cdot 8-22 \cdot 4$ & 5 & $2 I \cdot 3$ & $18 \cdot 2-26 \cdot 6$ \\
\hline 2 & 5 & $28 \cdot 3$ & $32 \cdot 2-33 \cdot 6$ & 5 & $26 \cdot 3$ & $21 \cdot 0-30 \cdot 8$ \\
\hline 3 & 4 & $23 \cdot 5$ & $23.0-37.8$ & 8 & $32 \cdot 1$ & $25 \cdot 2-37 \cdot 8$ \\
\hline 4 & 5 & $34 * 3$ & $32.2-35.0$ & 4 & $35 \cdot 3$ & $32 \cdot 2-39^{\prime 2}$ \\
\hline 5 & 6 & $43 \cdot 8$ & $39 \cdot 2-49 \cdot 0$ & 4 & $39^{\circ} 9$ & $36 \cdot 4-44 \cdot 8$ \\
\hline 6 & 4 & $44 \cdot 5$ & $37 \cdot 8-67 \cdot 2$ & 13 & $43 \cdot 7$ & $33 \cdot 6-54 \cdot 6$ \\
\hline 7 & 3 & $51 \cdot 8$ & $49 . \circ-54.6$ & 6 & $48 \cdot 4$ & $43 \cdot 4-58 \cdot 8$ \\
\hline$\dot{8}$ & 6 & $54 \cdot 6$ & $43 \cdot 4-67 \cdot 2$ & 3 & $55^{\circ} 0$ & $51 \cdot 8-57 \cdot 4$ \\
\hline 9 & 3 & 64.0 & $63 \cdot 0-64 \cdot 4$ & 6 & $58 \cdot 1$ & $50 \cdot 4-67 \cdot 2$ \\
\hline 10 & 8 & $67 \cdot 9$ & $58 \cdot 8-85 \cdot 4$ & 7 & 70.0 & $57.4-85.4$ \\
\hline I I & 6 & $79 \cdot 2$ & $60 \cdot 2-106 \cdot 4$ & II & $8 I \cdot I$ & $54 \cdot 6-114 \cdot 8$ \\
\hline 12 & 10 & 84.6 & $57.4-1 \times 6.2$ & 4 & $92 \cdot 0$ & $60.2-113.4$ \\
\hline 13 & 6 & $98 \cdot 5$ & $91 \cdot 0-106 \cdot 4$ & 8 & $102 \cdot 5$ & $79 \cdot 8-130 \cdot 2$ \\
\hline 14 & IO & 104.6 & $8 I \cdot 2-x 3 r \cdot 6$ & 5 & 102.5 & $85.4-130.2$ \\
\hline 15 & 9 & 123.3 & $100 \cdot 8-148.4$ & 2 & $116 \cdot 2$ & $102.2-130.2$ \\
\hline 16 & 4 & $140 \cdot 0$ & $121 \cdot 8-165 \cdot 2$ & 2 & $131 \cdot 6$ & $126 \cdot 0-137^{\circ} 2$ \\
\hline $17-20$ & 12 & 143.5 & $I 23 \cdot 2-I 72 \cdot 2$ & 9 & $137 \cdot 6$ & $100 \cdot 8-214.3$ \\
\hline $21-30$ & 17 & 159.3 & $128.8-191.8$ & 18 & $154^{\circ} \mathrm{I}$ & $100 \cdot 8-214.2$ \\
\hline $31-40$ & 29 & $172 \cdot 9$ & I I $4 \cdot 8-226 \cdot 8$ & 24 & $117 \cdot 2$ & $117 \cdot 6-225 t$ \\
\hline $41-50$ & 9 & $172 \cdot 5$ & $148 \cdot 4-240 \cdot 8$ & 10 & $170 \cdot 2$ & $105^{\circ} 0-253^{\circ} 4$ \\
\hline $51-60$ & 15 & $165 \cdot 1$ & $116 \cdot 2-252 \cdot 0$ & I 4 & $162 \cdot 5$ & I $13.4-235 \cdot 2$ \\
\hline Total & I75 & - & - & 180 & - & - \\
\hline $\begin{array}{l}20-50 y \\
20-50 y\end{array}$ & $\begin{array}{l}\text { ars (with ch } \\
\text { ars (without }\end{array}$ & $\begin{array}{l}\text { (dren) } \\
\text { children) }\end{array}$ & & $\begin{array}{r}48 \\
9\end{array}$ & $\begin{array}{l}167.4 \\
170.8\end{array}$ & $\begin{array}{l}113.4-225 \dagger \\
102.2-196\end{array}$ \\
\hline
\end{tabular}

\section{Laboratory tests}

Haemoglobin and erythrocyte count. The results of the haemoglobin determinations are shown in Table 12. The mean haemoglobin level with its standard error of all the subjects tested, both males and females, was $14.18 \pm 0.09 \mathrm{~g}$. $/ 100 \mathrm{ml}$. blood as measured colorimetrically. Calculated from the specific gravity of the blood, a mean level of $14.4 \mathrm{I} \pm 0.07 \mathrm{~g} / 100 \mathrm{ml}$. blood was obtained. Although the results by these two methods did not always coincide, there was a significant correlation of $r=0.475 \pm 0.045$ between these two means. There was a significant difference between males and females in the 16-45 age group, although the absolute values were normal. Erythrocyte counts on I 49 subjects gave a mean level of $4.55 \pm 0.07 \mathrm{million} / \mathrm{cu} . \mathrm{mm}$. The lowest reading found was 3 million cu.mm.

Plasma protein. The results of the determinations of plasma protein are shown in Table 13. The levels generally were high, and increased with age. This increase commenced early, as three blood samples from the youngest investigated-in the I-3 years age group--were over $6.5 \mathrm{~g} . / 100 \mathrm{ml}$.; although this was within generally accepted limits, there was a distinct upward trend with age. 


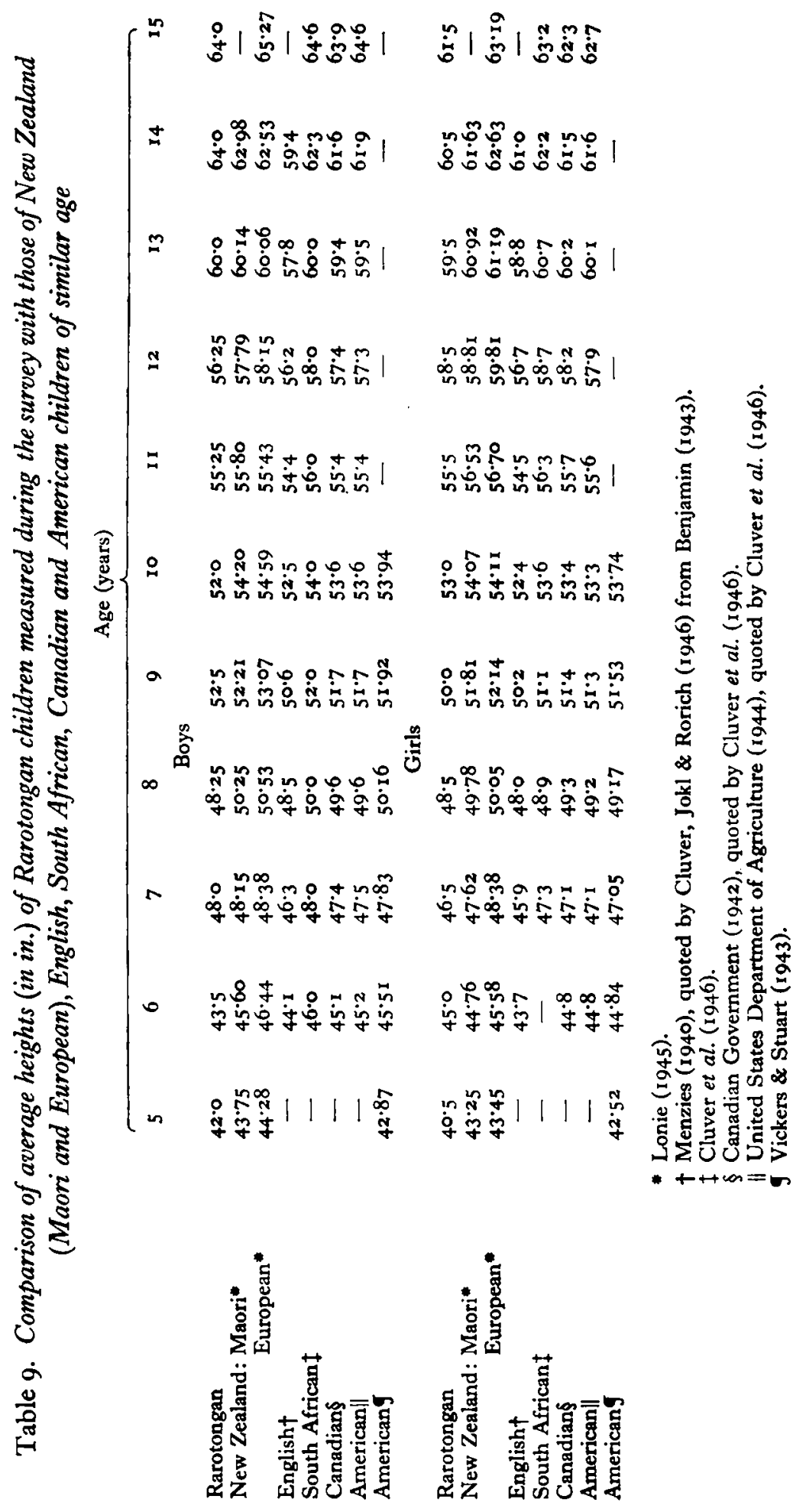




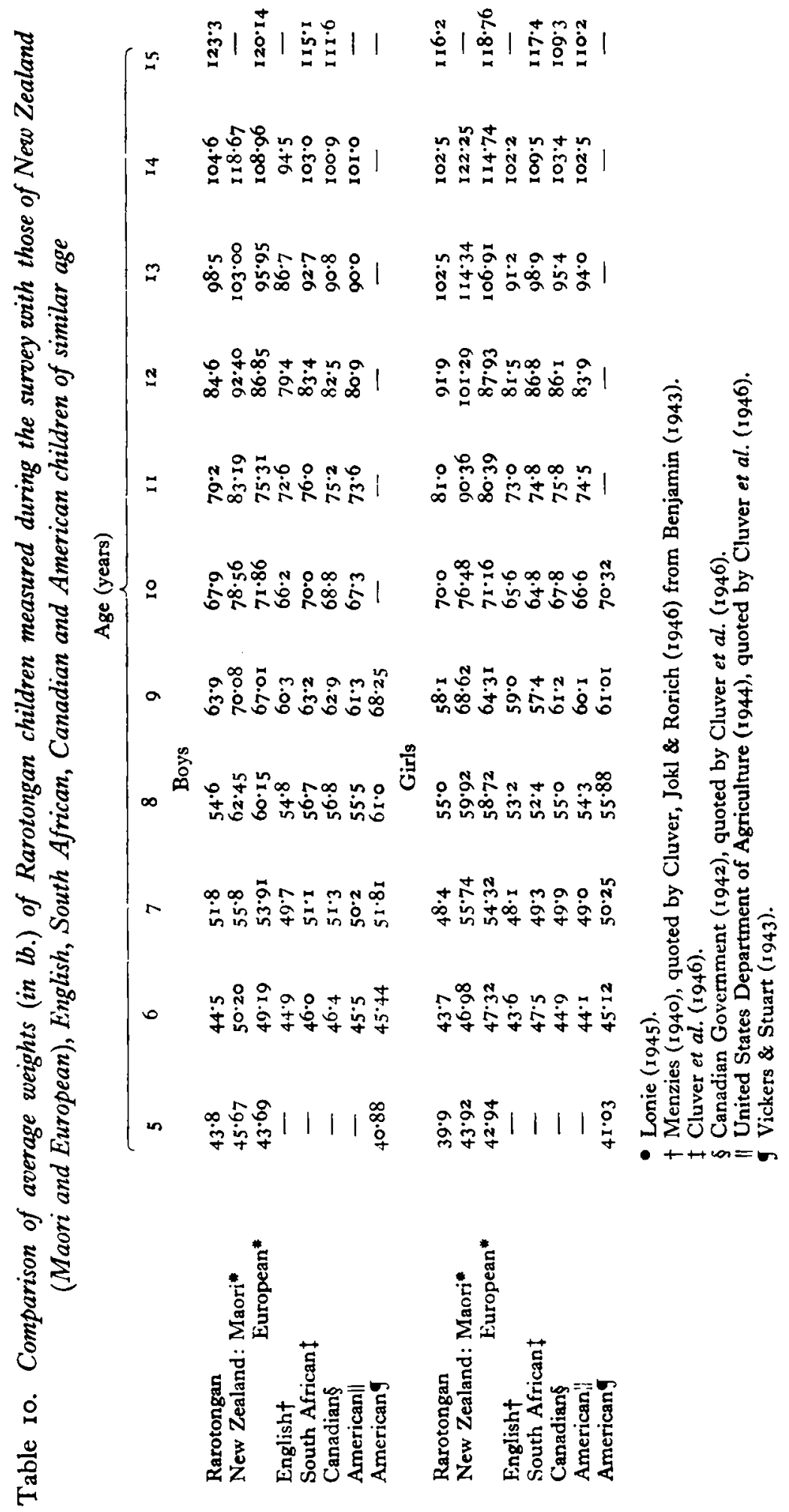


Table I I. Weight: height (W:H) ratio and Tuxford index $(T)$ of subjects of survey and others calculated from Tables 9 and $10 *$

\begin{tabular}{|c|c|c|c|c|c|c|c|c|c|c|c|c|}
\hline & \multicolumn{11}{|c|}{ Age (years) } \\
\hline & & 5 & 6 & 7 & $\begin{array}{c}8 \\
\text { Boys }\end{array}$ & 9 & 10 & II & 12 & 13 & 14 & 15 \\
\hline Rarotongan: & $\begin{array}{l}W: H \\
T\end{array}$ & $\begin{array}{l}1042 \\
1065\end{array}$ & $\begin{array}{r}1022 \\
999\end{array}$ & $\begin{array}{l}1079 \\
1007\end{array}$ & $\begin{array}{l}1132 \\
1006\end{array}$ & $\begin{array}{l}1217 \\
1027\end{array}$ & $\begin{array}{l}1305 \\
1044\end{array}$ & $\begin{array}{l}1433 \\
1082\end{array}$ & $\begin{array}{l}1504 \\
1069\end{array}$ & $\begin{array}{l}1641 \\
1094\end{array}$ & $\begin{array}{l}1643 \\
1017\end{array}$ & $\begin{array}{l}1926 \\
1113\end{array}$ \\
\hline $\begin{array}{l}\text { New Zealand } \\
\text { Maori: }\end{array}$ & $\begin{array}{l}W: H \\
T\end{array}$ & $\begin{array}{l}1043 \\
1066\end{array}$ & $\begin{array}{l}1100 \\
1075\end{array}$ & $\begin{array}{l}\text { I1 } 58 \\
1080\end{array}$ & $\begin{array}{l}1243 \\
1105\end{array}$ & $\begin{array}{l}\text { I } 342 \\
\text { I } 133\end{array}$ & $\begin{array}{l}1440 \\
1152\end{array}$ & $\begin{array}{l}1491 \\
1127\end{array}$ & $\begin{array}{l}1599 \\
1137\end{array}$ & $\begin{array}{l}1713 \\
1142\end{array}$ & $\begin{array}{l}1884 \\
\text { I } 172\end{array}$ & - \\
\hline $\begin{array}{c}\text { New Zealand } \\
\text { European: }\end{array}$ & $\begin{array}{l}W: H \\
T\end{array}$ & $\begin{array}{r}987 \\
1008\end{array}$ & $\begin{array}{l}1059 \\
1035\end{array}$ & $\begin{array}{l}1114 \\
1039\end{array}$ & $\begin{array}{l}\text { I } 190 \\
1057\end{array}$ & $\begin{array}{l}1263 \\
1066\end{array}$ & $\begin{array}{l}1316 \\
1052\end{array}$ & $\begin{array}{l}1358 \\
1026\end{array}$ & $\begin{array}{l}1493 \\
1062\end{array}$ & $\begin{array}{l}1597 \\
1065\end{array}$ & $\begin{array}{l}1742 \\
1084\end{array}$ & $\begin{array}{l}1841 \\
1064\end{array}$ \\
\hline English: & $\begin{array}{l}W: H \\
T\end{array}$ & $\begin{array}{l}971 \\
992\end{array}$ & $\begin{array}{r}1018 \\
995\end{array}$ & $\begin{array}{l}1073 \\
1001\end{array}$ & $\begin{array}{l}1129 \\
1004\end{array}$ & $\begin{array}{l}1191 \\
1006\end{array}$ & $\begin{array}{l}1260 \\
1008\end{array}$ & $\begin{array}{l}1334 \\
1008\end{array}$ & $\begin{array}{l}1413 \\
1005\end{array}$ & $\begin{array}{r}1500 \\
999\end{array}$ & $\begin{array}{r}\text { I } 59 \text { I } \\
989\end{array}$ & - \\
\hline South African: & $\begin{array}{l}W: H \\
T\end{array}$ & - & $\begin{array}{r}1000 \\
977\end{array}$ & $\begin{array}{r}1064 \\
993\end{array}$ & $\begin{array}{l}1134 \\
1008\end{array}$ & $\begin{array}{l}1215 \\
1026\end{array}$ & $\begin{array}{l}1296 \\
1037\end{array}$ & $\begin{array}{l}1357 \\
1025\end{array}$ & $\begin{array}{l}1438 \\
1023\end{array}$ & $\begin{array}{l}1545 \\
1029\end{array}$ & $\begin{array}{l}1653 \\
1028\end{array}$ & $\begin{array}{l}1782 \\
1029\end{array}$ \\
\hline Canadian: & $\begin{array}{l}W: H \\
T\end{array}$ & - & $\begin{array}{l}1028 \\
1005\end{array}$ & $\begin{array}{l}1082 \\
1009\end{array}$ & $\begin{array}{l}1145 \\
1018\end{array}$ & $\begin{array}{l}1217 \\
1028\end{array}$ & $\begin{array}{l}1284 \\
1027\end{array}$ & $\begin{array}{l}1357 \\
1025\end{array}$ & $\begin{array}{l}1437 \\
1022\end{array}$ & $\begin{array}{l}1528 \\
1019\end{array}$ & $\begin{array}{l}1637 \\
1018\end{array}$ & $\begin{array}{l}1746 \\
1009\end{array}$ \\
\hline American: & $\begin{array}{l}W: H \\
T\end{array}$ & $\begin{array}{l}954 \\
975\end{array}$ & $\begin{array}{r}1000 \\
977\end{array}$ & $\begin{array}{r}1057 \\
987\end{array}$ & $\begin{array}{r}\text { I I I I I } \\
988\end{array}$ & $\begin{array}{r}\mathbf{1} 8 \text { I } \\
997\end{array}$ & $\begin{array}{l}1255 \\
1004\end{array}$ & $\begin{array}{l}1329 \\
1004\end{array}$ & $\begin{array}{l}1411 \\
1003\end{array}$ & $\begin{array}{l}1503 \\
1002\end{array}$ & $\begin{array}{l}1631 \\
1015\end{array}$ & - \\
\hline & & & & & Girls & & & & & & & \\
\hline Rarotongan: & $\begin{array}{l}W: H \\
T\end{array}$ & $\begin{array}{r}985 \\
1039\end{array}$ & $\begin{array}{l}971 \\
975\end{array}$ & $\begin{array}{r}104 I \\
992\end{array}$ & $\begin{array}{l}\text { I I } 34 \\
\text { I023 }\end{array}$ & $\begin{array}{r}\text { I } 62 \\
988\end{array}$ & $\begin{array}{l}1321 \\
1057\end{array}$ & $\begin{array}{l}1359 \\
1093\end{array}$ & $\begin{array}{l}1571 \\
1096\end{array}$ & $\begin{array}{l}\text { I723 } \\
\text { II I } 4\end{array}$ & $\begin{array}{l}1694 \\
1009\end{array}$ & $\begin{array}{l}1889 \\
\text { 1029 }\end{array}$ \\
\hline $\begin{array}{l}\text { New Zealand } \\
\text { Maori: }\end{array}$ & $\begin{array}{l}W: H \\
T\end{array}$ & $\begin{array}{l}1015 \\
1071\end{array}$ & $\begin{array}{l}1049 \\
1053\end{array}$ & $\begin{array}{l}1170 \\
1115\end{array}$ & $\begin{array}{l}1204 \\
1086\end{array}$ & $\begin{array}{l}1324 \\
1127\end{array}$ & $\begin{array}{l}\text { I } 4 \text { I } 4 \\
\text { I I } 3 \text { I }\end{array}$ & $\begin{array}{l}\simeq 598 \\
I I 97\end{array}$ & $\begin{array}{l}1722 \\
1202\end{array}$ & $\begin{array}{l}1877 \\
1214\end{array}$ & $\begin{array}{l}1984 \\
1182\end{array}$ & - \\
\hline New Zealand & & & & & & & & & & & & \\
\hline European : & $\begin{array}{l}W: H \\
T\end{array}$ & $\begin{array}{r}988 \\
1043\end{array}$ & $\begin{array}{l}1038 \\
1042\end{array}$ & $\begin{array}{l}1123 \\
1070\end{array}$ & $\begin{array}{l}1173 \\
1058\end{array}$ & $\begin{array}{l}1233 \\
1049\end{array}$ & $\begin{array}{l}1315 \\
1052\end{array}$ & $\begin{array}{l}1418 \\
1062\end{array}$ & $\begin{array}{l}1470 \\
1026\end{array}$ & $\begin{array}{l}x 747 \\
1129\end{array}$ & $\begin{array}{l}1832 \\
1091\end{array}$ & $\begin{array}{l}1879 \\
1023\end{array}$ \\
\hline English: & $\begin{array}{l}W: H \\
T\end{array}$ & - & $\begin{array}{r}998 \\
1002\end{array}$ & $\begin{array}{r}1048 \\
999\end{array}$ & $\begin{array}{r}1108 \\
999\end{array}$ & $\begin{array}{r}175 \\
999\end{array}$ & $\begin{array}{l}1252 \\
1002\end{array}$ & $\begin{array}{l}1339 \\
1003\end{array}$ & $\begin{array}{l}1437 \\
1003\end{array}$ & $\begin{array}{l}1551 \\
1003\end{array}$ & $\begin{array}{r}675 \\
998\end{array}$ & - \\
\hline South African: & $W: H$ & 一 & - & 1042 & 1072 & I I 23 & 1208 & I328 & I 478 & 1629 & 1760 & 1857 \\
\hline Canadian: & $T$ & - & - & 993 & 967 & 956 & 966 & 995 & 1031 & 1054 & 1049 & 1011 \\
\hline Canadian: & $\begin{array}{l}W: H \\
T\end{array}$ & - & $\begin{array}{l}1002 \\
1006\end{array}$ & $\begin{array}{l}1059 \\
1009\end{array}$ & $\begin{array}{l}1116 \\
1006\end{array}$ & $\begin{array}{l}1191 \\
1014\end{array}$ & $\begin{array}{l}1269 \\
1015\end{array}$ & $\begin{array}{l}1361 \\
1019\end{array}$ & $\begin{array}{l}1479 \\
1032\end{array}$ & $\begin{array}{l}1584 \\
1025\end{array}$ & $\begin{array}{l}1081 \\
1001\end{array}$ & $\begin{array}{r}\mathbf{1} 754 \\
955\end{array}$ \\
\hline American: & $W: H$ & 964 & 995 & 1056 & I I 22 & $x 177$ & 1249 & I 340 & 1449 & 1564 & 1663 & I 757 \\
\hline & & 1017 & 999 & 1007 & 1012 & 1002 & 999 & $1 \infty 4$ & 1011 & 1012 & 991 & 957 \\
\hline
\end{tabular}

Table I2. Mean haemoglobin values determined colorimetrically on the subjects of the survey

\begin{tabular}{|c|c|c|c|c|c|}
\hline \multirow[b]{2}{*}{$\begin{array}{c}\text { Age } \\
\text { (years) }\end{array}$} & \multicolumn{2}{|r|}{ Males } & \multirow{2}{*}{$\begin{array}{l}\text { Significance of } \\
\text { difference between } \\
\text { males and females }\end{array}$} & \multicolumn{2}{|r|}{ Females } \\
\hline & $\begin{array}{l}\text { No. } \\
\text { examined }\end{array}$ & $\begin{array}{c}\text { Haemoglobin } \\
\text { (g. } / 100 \mathrm{ml} \text {. blood) }\end{array}$ & & $\begin{array}{c}\text { No. } \\
\text { examined }\end{array}$ & $\begin{array}{c}\text { Haemoglobin } \\
\text { (g./100 ml. blood })\end{array}$ \\
\hline $\begin{array}{l}\text { Under } 16 \\
16-45 \\
\text { Over } 45\end{array}$ & $\begin{array}{l}72 \\
63 \\
21\end{array}$ & $\begin{array}{l}13.85 \pm 0.19^{*} \\
15.29 \pm 0.14^{*} \\
15.0 \dagger\end{array}$ & $\begin{array}{l}\text { Not significant } \\
\text { Significant } \\
\text { Not significant }\end{array}$ & $\begin{array}{l}70 \\
58 \\
20\end{array}$ & $\begin{array}{l}14.25 \pm 0.1 .5 \\
14.30 \pm 0.22 \\
15.2 \dagger\end{array}$ \\
\hline Total & 156 & $14.57+0.12^{\circ}$ & Significant & 148 & $13.83 \pm 0.12$ \\
\hline
\end{tabular}

- Value with its standard error.

+ No. of cases examined considered too small to provide a valid estimate of error. 
Table 13. Mean plasma-protein values determined on subjects of the survey

\begin{tabular}{|c|c|c|}
\hline $\begin{array}{c}\text { Age } \\
\text { (years) }\end{array}$ & $\begin{array}{l}\text { No. } \\
\text { examined }\end{array}$ & $\begin{array}{l}\text { Plasma protein } \\
\text { (g./100 ml. })\end{array}$ \\
\hline $0-6$ & 29 & $7 \cdot 88 \pm 0 \cdot 17$ \\
\hline $\begin{array}{c}7-15 \\
16 \text { and over }\end{array}$ & $\begin{array}{l}110 \\
151\end{array}$ & $\left.\begin{array}{l}8.43 \pm 0.09 \\
8.72 \pm 0.08\end{array}\right\}$ Difference significant \\
\hline Total & 290 & $8 \cdot 51 \pm 0.06$ \\
\hline
\end{tabular}

Sedimentation rate. The sedimentation rate was measured in 290 people. If normal limits are taken as $5 \mathrm{~mm}$. $/ \mathrm{hr}$. for men and $15 \mathrm{~mm}$. $/ \mathrm{hr}$. for women, then the sedimentation rate must be considered abnormally elevated in $67 \%$ of the subjects. In $20 \%$ it was over $30 \mathrm{~mm} . / \mathrm{hr}$. In three cases it was over $50 \mathrm{~mm}$. $/ \mathrm{hr}$. In I2I cases with a sedimentation rate of under $15 \mathrm{~mm}$. $/ \mathrm{hr}$., the mean plasma protein was $8.38 \pm 0.082 \mathrm{~g}$. $100 \mathrm{ml}$., whereas in 160 cases with a sedimentation rate of over $15 \mathrm{~mm}$. $/ \mathrm{hr}$., the mean plasma protein was $8.6 \mathrm{I} \pm 0.72 \mathrm{~g} / 100 \mathrm{ml}$. This difference shows a probability of $30: \mathrm{I}$ and is taken as significant. The explanation of this observation is considered in the discussion.

\section{Dietary assessment}

Questions were asked in each house as to the times when food was eaten and the nature of the food consumed. It was found that, whereas the staple food still remains kumara, taro, breadfruit, arrowroot and green bananas, increasing dependence is being placed on tinned food bought from the stores, of which there were eight in the village of Arorangi alone. About one-third of the total imports to the Cook Island group are foodstuffs, mainly tinned meat, flour, sugar and biscuits. In the Annual Report of the Cook Islands for the year ended 3 I March 1949, foodstuffs were given as $32.5 \%\left(£ 97,45^{\circ}\right)$ of the total imports (New Zealand Government, 1950). In few families was any milk consumed, and then it was reserved for babies or sick people. Goat's milk was in occasional use. Green vegetables were scarce. Butter, cheese and eggs were rarely eaten. Coconut in some form, particularly as coconut cream, was used in all the families every day. It was surprising to find that fish and fish foods bulked largely in the dietary, despite many statements to the contrary. Each family seemed to collect its own sea-food products. In only one household was flour bought as such.

Milk in schools. The introduction of a milk ration in all the schools in Rarotonga had been in operation for 18 months and the teachers were enthusiastic as to its advantages.

Meals. In every instance breakfast was a very sketchy meal. In some no breakfast at all was eaten. In the remainder it consisted of bread without butter and tea without milk. In only three instances was animal protein eaten at this meal. Frequently the children went to school without breakfast. In all the families, the principal meal of the day was eaten at midday. Fresh meat was a luxury and, when eaten, consisted principally of pork. The evening meal was very similar to the breakfast, consisting chiefly of 
bread and tea. Local fruits, such as tomatoes and citrus fruits, were eaten freely when in season, but the pawpaw-rich in vitamin $\mathrm{C}$ and a good source of carotene and so attractive in its flavour and texture to the European-was eaten only by babies and pigs.

\section{Vital statistics}

Infant-mortality figures have been reported reliably since the introduction of death certification by Cook Islands medical practitioners in 1947. In 1949-50 there were seventy infant deaths and 621 births, giving an infant mortality rate of $\mathrm{I}_{13} / 1000$ live births (New Zealand Government, 1950). During 1948-9 only one death from each of marasmus, debility and malnutrition was recorded throughout the whole Cook Islands group (New Zealand Government, 1949). No deaths directly attributable to malnutrition were noted in 1949-50 in Rarotonga. Although deaths in the rst year of life accounted for $26.3 \%$ of total deaths in $1948-9$ and $23.25 \%$ in $1949-50$, infections were the greatest cause of death $(35 \%$ in the whole islands in $1948-9$, and $60 \%$ in Rarotonga in 1949-50). Mortality in the 2nd year of life was $4-5 \%$ of total deaths. Half the population of Rarotonga, which has doubled since 1906 , consists of children (Table I).

\section{DISCUSSION}

Nutritional status may be assessed by (a) considering the quantity and quality of foodstuffs consumed, and $(b)$ the results of a clinical examination and laboratory tests designed to discover nutritional disorders.

Davis (1947), Abraham (1947), Peren (1947) and Wingfield (1949) all comment on the change from native to European foods; our findings amply confirm this. While native foods take a minor place, the Rarotongan depends largely upon imported tinned meat, supplemented by fish and shell-fish which are becoming inadequate in supply, for his chief protein sources; all of these are limited by cost or availability. This state of affairs is, in turn, a reflexion on the state of animal husbandry on the island. Very few cows, all in apparently poor condition, are kept; goats and pigs are common domestic animals and most families keep poultry, but there is no attempt at scientific farming either for meat or eggs.

Figures available for infant mortality in both Rarotonga and the whole Cook Islands group, show that infection rather than frank malnutrition is the chief cause of death. How much the high infection rate may be influenced by suboptimal nutrition is not known; infective conditions will be discussed separately in a subsequent publication (Faine \& Hercus, I951).

Protein deficiency, if present, is not reflected either in the plasma proteins or in clinical signs. Indeed, the plasma proteins are relatively high, comparable to figures found for serum by Wills \& Bell (195I) in Fiji and Samoa, using the same coppersulphate method and the same formula for calculation from the specific gravity. A sample of serum submitted to electrophoretic fractionation by these workers showed a twofold increase in the $\gamma$-globulin fraction. The increase in plasma protein in Rarotongans is similar, both in magnitude and in age distribution, to the increase in the serums of Fijians and Samoans. Also the association of raised plasma protein 
with a rapid sedimentation rate in the Rarotongans suggests that it may be related to some infection.

The results of haemoglobin determinations and of erythrocyte counts showed no signs of iron deficiency, nor were any serious cases of anaemia found, even among women of reproductive age, many of whom had large families.

Clinical examination, substantiated by laboratory findings, showed very little 'malnutrition'. Pollack (1950), in a review of the physical signs of nutritional disturbance, points out that the degree of abnormality required for the diagnosis of nutritional disturbance varies with the observer and with his experience of nutritional disorders. Nevertheless, none of the signs he considers indicative or suspicious of nutritional disturbance were seen, so that the problem of the interpretation of signs noted does not arise. A clinical or laboratory search for signs of malnutrition is, at the best, a negative approach to the problem of assessment of nutritional status. It is more important to be able to credit for 'good' nutrition than to debit for 'malnutrition'. The 'subjective nutritional assessment' contributes to the positive side to some extent. Here the difficulty is not deduction of marks for 'malnutrition' but how to assess obesity, which was common. If obesity is to be considered a state of nutritional disturbance, as it is, then marks must be deducted; if this is done there is no way of distinguishing between the obese and the undernourished. However, if this distinction is not made, there is still a difference between those considered to be of good nutrition and those of unsatisfactory nutrition, whether the latter state be due to undernourishment or obesity. Here marks have been deducted for obesity.

Obesity, as seen from the height-weight graph, is an important problem, especially among the women, very few of whom are tall in proportion to their weights. A dietary factor that alone could be responsible was not obvious, unless it could be the apparent reliance placed on foods in the diet containing carbohydrate and fat. Diabetes is said to be rare. Perhaps there is a racial factor, possibly of dietary habit, as this obesity appears to be common to several Polynesian groups, especially among the women. Many workers have attempted to provide some index, readily arrived at from a few simple measurements, that would give an objective measurement of nutritional status. Of nearly all of these methods, reviewed by Tuxford (1939), Stuart \& Meredith (1946) and Stevenson (1950), height and weight are the fundamental factors, which are corrected for age by some when dealing with children. There is little doubt that physique reflects nutritional disturbances, so that measurement of physique may be taken as a measure of a positive aspect of nutritional status, in the absence of gross organic factors affecting physique. Height and weight are both gained progressively during childhood and adolescence, but there is a wide range of normal values in each age group due to individual variations in body-type, sexual maturity and racial factors. For these reasons ratios or indices based on height and weight have been recommended as a more constant measure of the individual's physique, irrespective of age. On the other hand, since the ratio of weight to height varies with age in normal children, it seems desirable that age be taken into consideration. Attempts have been made to standardize this factor in various ways. Tuxford $(1939,1942)$ has recommended the use of a formula which reduces the curved $W: H$ graph to a straight line, along an 'index' 
of 1000 in London schoolchildren. Wetzel (1941), modifying the $W: H$ ratio for age and body type, has developed an index which is excellent for following individual cases in successive examinations, but is of little significance for single observations, as in this survey. Here we have preferred to use the 'Tuxford index, which means that a child with a higher index is heavier for its height, than other comparable children, regardless of age. It will be seen from Fig. 2 that the Tuxford index is approximately 1000 only for English, North American and Canadian children, whereas Rarotongan, South African and New Zealand children, both Maori and European, vary considerably from this level. Recently published figures show that with an increase in average heights and weights in London children (Daley, 1950), there was only a slight increase in Tuxford's index, especially for girls, since 1938 .

These considerations emphasize the reflexion of physique in the Tuxford-index score, and the value of some such ratio for providing a base-line for comparison between groups. It is obvious, however, that a different manipulation of the $W: H$ ratio for age must be made to allow for racial or local variations, as with New Zealand Maoris, for example, to give an index of 1000 . It will also be noted from Fig. 2 that an endocrine factor probably plays some part in determining the differences between groups, as the variation from 1000 is most marked in each group among girls from Io to 15 years old.

The results show that the physique of Rarotongan children and adults compares favourably with that of people in other parts of the world, and that it does not reflect signs of malnutrition.

Comparison of the heights and weights of Rarotongan children with figures for children of other countries (Meredith, 1948; Barach, 1945) substantiates this.

Dental conditions in Rarotonga have been reported by Wingfield (1949), who found that the incidence of dental caries in schoolchildren averaged eight teeth per child, two-thirds of the cavities being in the early stages of decay (stages I and 2) and onethird in advanced stages (stages 3 and 4). An examination of 500 adults showed also eight carious teeth per head, and abnormal gum conditions in $48 \%$. The findings in this survey show an almost universal incidence of caries with some increase in degree with age. Also, there is a steady increase with age in the number of teeth missing and a general picture of absence of prevention or treatment of dental disease. The only people with teeth filled are those who are young enough to have come under the influence of the School Dental Service set up a few years ago; it is from this end that the problem is being tackled. The state of the gums, though most unsatisfactory, compares favourably with the findings in Samoa by the 1948-9 expedition (unpublished report to New Zealand Medical Research Council). To what extent the hypoplasia of the enamel found in some cases, or the large amount of caries and gingivitis, comes from a nutritional background, is difficult to tell. There is no evidence for or against calcium deficiency, which is not believed to be present. There is almost certainly a relationship to the introduction of European foods, as it has been recorded by several observers that the natives' teeth were in excellent condition many years ago. Also, inhabitants of Rarotonga, some of them among the group studied, tell how their teeth were free from dental caries on other islands where they lived until they moved to Rarotonga; 
here they consumed a much larger amount of European food, especially soft carbohydrates, whereas on the other Cook islands they had to exist on hard native foods.

In the absence of other signs of nutritional deficiency, which might be expected if these oral changes found represented a lack of essential dietary components, it might be concluded that the problem is primarily an oral one, and could be remedied by education in oral hygiene, efficient prophylaxis and treatment in the early stages of dental caries.

It was of considerable interest to note that there was only one mild case of thyroid enlargement; the large quantity of fish and sea-foods consumed must provide a relatively large iodine intake.

From all these findings, it will be seen that the diet must essentially be adequate in quality and quantity. Inasmuch as the economic status of the individual governs the amount and type of food he consumes, so long as he relies (especially for animal protein) upon imported food that must be bought, the diet could be improved by better planning of local food production and by the fostering of animal and poultry husbandry.

\section{SUMMARY}

I. A representative sample of Rarotongans has been investigated by clinical and laboratory methods and their nutritional status assessed.

2. The results showed that malnutrition is neither a widespread nor a serious problem except that the influence of European food apparently plays an important part in the very common unhealthy state of the teeth and mouth.

3. The diets were apparently sufficient in protein, carbohydrate, fat, minerals and vitamins.

4. Obesity was common, due possibly to racial as well as dietary factors. 'The physique of both adults and children compared favourably with that of similar groups in other countries.

We wish to thank the New Zealand Government Department of Island Territories, the New Zealand Medical Research Council, the Cook Islands Administration, Dr T. R. A. Davis and numerous others for their assistance and co-operation; also Mr J. A. Samuel for his technical assistance.

\section{REFERENCES}

Abraham, M. (1947). Report on Food Conditions in Rarotonga. Report to Department of Island Territories, N.Z. Government.

Barach, J. H. (1945). Amer. Y. Dis. Child. 69, 92.

Benjamin, B. (1943). F. Hyg., Camb., 43, 55.

Canadian Government (1942). A Height and Weight Survey of Toronto Elementary Schoolchildren. Ottawa: Government Printer.

Cluver, E. H., Jokl, E. \& Rorich, R. R. (1946). S. Afr. F. med. Sci. rr, 45.

Daley, A. (1950). Report on the Heights and Weights of School Pupils in the County of London in 1949, pp. 4 and 14. London: I-ondon County Council.

Davis, T. R. A. (1947). F. Polynesian Soc. 56, 197.

Faine, S. \& Hercus, C. E. (1951). Trans. R. Soc. trop. med. Hyg. (In the Press.)

Hoch, H. \& Marrack, J. (1945). Brit. med. F. ii, I 5 I.

Lonie, T. C. (1945). N.Z. med. F. 44, I69. 
Mellanby, M. (1934). Spec. Rep. Ser. med. Res. Coun., Lond., no. 191.

Menzies, F. (1940). Report by the School Medical Officer on the Average Heights and Weights of Elementary School Children, p. 22. London: London County Council.

Meredith, H. V. (1948). Child Developm. r9, I79.

New Zealand Government (1949). Annual Report on Cook Islands, p. I4. Department of Island Territories, N.Z. Government, 1948-49.

New Zealand Government (1950). Annual Report on Cook Islands, p. I2. Department of Island Territories, N.Z. Government, r949-50.

Peren, G. S. (1947). Bull. Massey agric. Col. no. 20.

Phillips, R. A., Van Slyke, D. D., Dole, V. P., Emerson, K. Jr., Hamilton, P. B. \& Archibald, R. M. (1945). Copper Sulfate Method for Measuring Specific Gravities of Whole Blood and Plasma. New York: Josiah Macy Jr. Foundation.

Pollack, H. (1950). Y. Mt Sinai Hosp. 16, 379.

Stevenson, A. C. (1950). Recent adv. soc. Med. I, I.

Stuart, H. C. \& Meredith, H. V. (1946). Amer. F. publ. Hlth, 36, 1365.

Tuxford, A. W. (1939). f. Hyg., Camb., 39, 203.

Tuxford, A. W. (1942). F. Hyg., Camb., 42, 549.

United States Department of Agriculture (1944). Misc. Publ. U.S. Dep. Agric. no. 366.

Van Slyke, D. D., Hiller, A., Phillips, R. A., Hamilton, P. B., Dole, V. P., Archibald, R. M. \& Eder, H. A. (1950). F. biol. Chem. 183, 33 r.

Van Slyke, D. D., Phillips, R. A., Dole, V. P., Hamilton, P. B., Archibald, R. M. \& Plazin, J. (1950). Y. biol. Chem. $183,349$.

Vickers, V. S. \& Stuart, H. C. (1943). F. Pediat. 22, 155.

Wetzel, N. C. (1941). F. Amer. med. Ass. x16, 1187.

Wills, I. \& Bell, M. E. (195I). Lancet, 260, 820 .

Wingfield, G. E. (1949). Report to the Seventh Pacific Science Congress, 1949. (In the Press.)

Wintrobe, M. M. (1935). Amer. F. med. Sci. 189, 102.

\title{
The Nutritive Value of Colostrum for the Calf
}

\section{The Effect of Prepartum Milking}

\author{
By R. ASCHAFFENBURG, S. BARTLETT, S. K. KON, J. H. B. ROY \\ AND D. M. WALKER \\ National Institute for Research in Dairying, University of Reading \\ AND C. BRIGGS AND R. LOVELL \\ Department of Pathology, Royal Veterinary College, London, N.W. r
}

(Received 4 Yanuary I95I)

The milking of cows and heifers before parturition has been advocated as a means of reducing the congestion of the udder and the associated oedema at calving, and of increasing the milk yield of the cow during the subsequent lactation period. The extent of pre-milking varies widely; on some farms, cows are milked only once or twice before parturition, whereas on others they are milked twice daily for up to 30 days.

The composition of the secretion produced at calving is altered by prepartum milking (Eaton, Johnson, Spielman, Matterson \& Nezvesky, 1949a), there being a reduction in the total nitrogen and in the percentage of non-casein nitrogen; the extent of this decline depends on the quantity of secretion removed before calving (VanLandingham, Weakley, Ackerman \& Hyatt, 1949). It is possible that the postpartum secretion of pre-milked cows may be of less benefit to newborn calves than normal colostrum, and it 